\title{
Atendimento odontológico humanizado à comunidade transexual: revisão de literatura
}

\author{
Humanized dental care for the transsexual community: literature review
}

Atención dental humanizada para la comunidad transexual: revisión de la literatura

Anthero Wesley Rodrigues da Silva Filho ORCID: https://orcid.org/0000-0001-8067-8177 Centro Universitário Cesmac, Brasil E-mail: anthero_wesley@outlook.com

Mayane Karyne Amâncio da Silva ORCID: https://orcid.org/0000-0001-7371-8812 Centro Universitário Cesmac, Brasil E-mail:mk-amancio@ hotmail.com

Géssyca Luyse Procópio Gonzaga ORCID: https://orcid.org/0000-0003-2111-5459 Centro Universitário Cesmac, Brasil E-mail: g.luyse@ hotmail.com

João de Deus Santos Neto ORCID: https://orcid.org/0000-0001-8442-0085 Centro Universitário Cesmac, Brasil E-mail: jd.sn68@gmail.com

Gabrielly Maria Argolo Aciolly ORCID: https://orcid.org/0000-0003-4248-7892 Centro Universitário Cesmac, Brasil E-mail: gaabiargolo@hotmail.com

Arthur Ravel Teles de Lima Bezerra ORCID: https://orcid.org/0000-0001-7096-7899 Centro Universitário Cesmac, Brasil E-mail: arthurravel@hotmail.com

Patricia Barbosa de Medeiros Melo ORCID: https://orcid.org/0000-0002-5054-1468 Centro Universitário Cesmac, Brasil

E-mail: patriciamedeiros_@outlook.com Letícia Braga Peixoto ORCID: https://orcid.org/0000-0001-9754-356X Centro Universitário Cesmac, Brasil

E-mail: peixoto.lec@gmail.com

Ana Luiza Pontes de Oliveira

ORCID: https://orcid.org/0000-0003-4744-0655 Centro Universitário Cesmac, Brasil E-mail: ana_luizapontes@hotmail.com Fernanda Braga Peixoto

ORCID: https://orcid.org/0000-0003-4549-4959 Centro Universitário Cesmac, Brasil

E-mail: fernandapeixoto_al@hotmail.com

\begin{abstract}
Resumo
Objetivo: Destacar e analisar como ocorre o atendimento odontológico humanizado da população transexual e identificar o conhecimento que o profissional Cirurgião-dentista possui a respeito das políticas públicas de atenção à essa população. Metodologia: Trata-se de um estudo de revisão narrativa da literatura. Revisão bibliográfica: É importante ressaltar a ausência de travestis e transexuais nos serviços de saúde, principalmente na atenção básica, que deveria ser a porta de entrada dos serviços de saúde. Quanto ao atendimento a gays, lésbicas, bissexuais, travestis e transexuais, as violações de direitos humanos básicos por parte de diversos grupos sociais (inclusive profissionais de saúde) como segregação, preconceito, marginalização social, etc., promovem a formulação de criar políticas públicas de saúde específicas para esses públicos para garantir a equidade no atendimento. Portanto, Como profissional de saúde, o cirurgião-dentista, além de conhecimentos técnicos, deve ter conhecimento da realidade social e do cuidado humanitário para realizar observações de forma multiprofissional. Considerações finais: Conclui-se que deve ter prestação de serviço digno, além de ser essencial um acesso a saúde ampliado fazendo-se necessário um investimento na preparação dos profissionais, para os tratamentos e cuidados específicos à comunidade transexual.
\end{abstract}

Palavras-chave: Transexualidade; Transexuais; Sexo; Atendimento odontológico. 


\begin{abstract}
Objective: To highlight and analyze how the humanized dental care of the transsexual population occurs and to identify the knowledge that the dental surgeon has about public policies for this population. Methodology: This is a study of narrative literature review. Literature review: It is important to emphasize the absence of transvestites and transsexuals in health services, especially in primary care, which should be the gateway to health services. As for the assistance to gays, lesbians, bisexuals, transvestites and transsexuals, violations of basic human rights by various social groups (including health professionals) such as segregation, prejudice, social marginalization, etc., promote the formulation of creating public policies specific health services for these audiences to ensure equity in care. Final Considerations: It is concluded that there must be a dignified service, making it necessary to invest in the preparation of professionals for specific treatments and care for the transsexual community.
\end{abstract}

Keywords: Transsexuality; Transsexuals; Sex; Dental care.

\title{
Resumen
}

Objetivo: Resaltar y analizar cómo se da la atención odontológica humanizada de la población transexual e identificar el conocimiento que tiene el cirujano dentista sobre las políticas públicas para esta población. Metodología: Se trata de un estudio de revisión de literatura narrativa. Revisión de la literatura: Es importante destacar la ausencia de travestis y transexuales en los servicios de salud, especialmente en la atención primaria, que debe ser la puerta de entrada a los servicios de salud. En cuanto a la asistencia a gays, lesbianas, bisexuales, travestis y transexuales, las violaciones de los derechos humanos básicos por parte de diversos grupos sociales (incluidos los profesionales de la salud) como la segregación, los prejuicios, la marginación social, etc., promueven la formulación de políticas públicas de salud específicas. Servicios para estos públicos para garantizar la equidad en la atención. Consideraciones finales: Se concluye que debe existir un servicio digno, por lo que es necesario invertir en la preparación de los profesionales para tratamientos y cuidados específicos para la comunidad transexual.

Palabras clave: Transexualidad; Transexuales; Sexo; Cuidado dental.

\section{Introdução}

A sexualidade humana é explorada dentro dos contextos biológicos, de gênero e de orientação sexual, ela intervém em diversos fatores na formação do indivíduo, por exemplo na subjetividade do ser, na relação consigo mesmo e com os demais modificando, portanto, o modo de se relacionar com a sociedade. A sexualidade é um instrumento organizador da personalidade, unificando os pilares biológicos, psicológicos e sociais (Albuquerque, et al., 2013).

Com isso, é importante perceber que identidade de gênero é diferente de sexo biológico. O sexo biológico condiz com a diferenças biológicas naturais do corpo (cromossomos, perfil hormonal, órgãos sexuais internos e externos), já a identidade de gênero se refere a uma construção social e cultural das diferenças sexuais, ou seja, a construção do que seriam as características masculinas e femininas (Silva, 2019).

Logo, nós podemos fazer uma separação de dois grupos: o cis gênero, nomenclatura a qual representa os indivíduos que não possuem divergência com o sexo atribuído no nascimento; e o trans gênero, que representa os indivíduos que possuem divergência com o sexo atribuído no nascimento. Lembrando sempre que para ser transgênero não necessariamente a pessoa precisa passar pelo processo de transgenitalização (intervenção cirúrgica para mudança do sexo biológico), essa só depende do desejo pessoal de readequar o seu corpo e sua genitália ao gênero com o qual se identifica (Rocon, et al., 2020).

Foi no ano de 1920 que os primeiros procedimentos de transgenitalização foram feitos na Alemanha e Dinamarca, onde as cirurgias genitais eram consideradas uma readequação social. Elas eram procedimentos feitos sem a divulgação para a sociedade, mas em 1952 foi realizada a cirurgia em um soldado americano e o assunto só então ganhou proporção no meio social. Esse caso trouxe bastante discussão nas áreas médicas sobre quais seriam os meios de tratamento e pouco tempo depois o sexólogo alemão Harry Benjamin sentiu a necessidade de criar o termo "transexualismo" que foi a primeira palavra para definir a condição e enquadrar indivíduos transexuais em um grupo só (Arán, 2006).

Porém na década de 40 os estudos com transexuais continuavam sendo feitos sem nenhuma base teórica, apenas com um entendimento do que era relatado pelos próprios pacientes e analisado pelos médicos. A palavra Transexual surgiu na década de 50, para aquelas pessoas que se identificam com o sexo oposto, não importando com hormônios, cirurgias ou qualquer tipo de alteração na fisionomia dessas pessoas. Já nas décadas de 60 e 70, o termo "transexual” passou a ser usado 
para aqueles que se sentiriam melhor após a cirurgia de mudança de sexo, eis então que surge o termo "síndrome de distrofia de gênero", que nada mais é do que o desconforto persistente com características sexuais atribuídas com o sexo de nascimento, justificando um tipo de distúrbio com os transexuais (Athayde, 2001). Lembrando que nem todos os transgêneros possuem essa síndrome.

Desde a década de 70, os brasileiros transexuais dispõem de meios cirúrgicos para a alteração de seu sexo natural, adequando sua situação física à sua situação emocional e psíquica, como alguém do sexo oposto. Porém, o reconhecimento a tais direitos não veio de imediato, sendo objeto de uma longa construção que ainda não se encontra finalizada. (Baracho, et al., 2010)

No Brasil, no ano de 1971, que foi feita a primeira cirurgia de transgenitalização pelo médico Roberto Farina. Mesmo a cirurgia ter sido realizada com sucesso, ele sofreu alguns processos na justiça com a justificativa de que a cirurgia teria sido mutiladora, indo contra o Código Penal e o de Ética Médica daquele ano (Saadeh, 2004).

Em 1997, com a mudança da legislação médica, o Brasil passou a realizar os procedimentos cirúrgicos de mudança de sexo, só assim o Conselho Federal de Medicina regulamentou as práticas cirúrgicas em transexuais, porém essas somente poderiam ser realizadas em hospitais universitários e alguns públicos que realizavam pesquisas na área. Já no ano de 2002, uma nova resolução revogou a anterior, a qual que para realizar o procedimento cirúrgico, o paciente teria que passar por um tratamento de no mínimo dois anos, tendo um acompanhamento com profissionais como psiquiatra, psicólogo e até assistente social (Arán, et al., 2009), para assim comprovar sua identidade da pessoa trans e evitar que a cirurgia causasse qualquer tipo de arrependimento futuro.

Hoje em dia saber o que alguns termos, conceitos e seus significados representam é indispensável para que haja respeito e não cause constrangimento durante o atendimento odontológico.

Segundo Trindade (2019) sexo biológico, é o que todo mundo sabe, são algumas características de anatomia e funcionamento do organismo. Identidade de gênero, é a identidade ou o sentimento de uma pessoa pertencer ao determinado gênero, masculino, feminino, a ambos ou a nenhum deles. Orientação sexual, é por quem ou por qual gênero masculino ou feminino a pessoa se atrai, sente algum afeto ou desejo de se relacionar. Quando nascemos é nos dado um gênero em nossos documentos homem ou mulher, que vão ditar o nosso funcionamento na sociedade, esse gênero e baseado no nosso sexo biológico ou nos nosso genitais. Cisgênero, é quando uma pessoa se identifica com o gênero que lhe foi designado ao nascimento ela é chamada cisgênera, por exemplo é o homem que se identifica com o gênero masculino e a mulher que se identifica com o gênero feminino. Transexual, é aquela que não se identifica com o gênero que lhe foi designado ao seu nascimento, por exemplo tendo características de um corpo masculino essa pessoa de identifica com o gênero feminino, por tanto essa pessoa é uma mulher mas sendo com o corpo biologicamente masculino o mesmo vale para pessoas que nasceram com o corpo com características femininas mas se identifica com o gênero masculino, se identifica por tanto como homem.

Entende-se como homem transexual, aquele que foi designado mulher ao nascer, porém, se identifica como sendo do sexo e gênero masculino. Já a mulher transexual é aquela que foi designada homem do nascimento, contudo, se identifica como sendo do sexo e gênero feminino (Jesus, 2012).

Embora esse grupo faça parte da sociedade e tenha diversas leis a seu favor, os transgêneros sofrem exclusões sociais diárias. Essas pessoas sofrem discriminação e preconceito em várias parte da vida, tanto pessoal, quanto social e profissional, e com o serviço de saúde, infelizmente, não é diferente. O mercado da saúde apresenta dificuldades de acesso a transgêneros, principalmente, devido a atendimentos discriminatórios, seguido por condutas inadequadas, ofensas e constrangimentos gerados pelo profissional da saúde (Santos, et al., 2020).

Além de que, ainda existe o problema da invisibilidade desse grupo social. Muitos profissionais da saúde afirmam a quantidade de pessoas LGBT que procuram atendimento médico é pequena, e dizem também que muitos não se assumem nas consultas, 
mostrando que grande parte dessa população não se sente confortável nas buscas de auxílio a saúde. Sendo comum a fala retirada da pesquisa de Guimarães (2017) onde os profissionais afirmam que não tem noção das problemáticas enfrentadas pelo grupo, e que não acham muito relevante já que quando eles buscam ajuda médica são tratados como "pacientes normais". Ou seja, apesar dos LGBT sofrerem diversos preconceitos, esse tema ainda não é muito abordado e difundido dentro da comunidade da saúde, e ainda existe o conceito preconceituoso de forma imperceptível nos imaginários dos profissionais de anormalidade quando os pacientes fogem do padrão heteronormativo.

Porém, apesar de tudo isso a população LGBT, em todas as fases da vida, tem direito à saúde integral, humanizada e de qualidade como qualquer outro usuário do SUS. Visto que a Portaria $\mathrm{n}^{\circ} 2.836$ de $1^{\circ}$ de dezembro de 2011 institui a Política Nacional de Saúde Integral LGBT, garantindo o atendimento integral, nas diversas formas de vivenciar a sexualidade, contribuindo para que esses usuários se sintam acolhidos nos serviços de saúde (Brasil, 2011).

De acordo com a portaria N. 1.829, de 13 de agosto de 2009, é assegurado às pessoas transgênero e travestis, dentro do SUS, o uso do nome social no acesso inicial e a tratamentos específicos para o processo transsexualizador. No que abrange o atendimento odontológico pelo SUS, o uso do nome social é de extrema relevância, desde o primeiro contato com o profissional até o fim do tratamento, pois o uso do nome social melhora não só o acolhimento dessa população pelo sistema, mas, também, estimula a procura e o acesso dessa população, aumentando a responsabilidade e o grau de vínculo e confiança entre profissional e usuários, estabelecendo uma interação humanizada e cidadã. Esses profissionais devem sempre estar preparados para atender e acolher a todos sem distinção de gênero, orientação sexual ou sexo biológico (Brasil, 2017).

O objetivo desse estudo é destacar e analisar como ocorre o atendimento odontológico humanizado da população transexual e identificar o conhecimento que o profissional Cirurgião-dentista possui a respeito das políticas públicas de atenção à essa população.

\section{Metodologia}

Trata-se de um estudo de revisão narrativa da literatura.

Para realizar este levantamento bibliográfico foi coletado estudos do ano de 2000 à 2020, incluiu-se pesquisas em bases eletrônicas e busca manual de citações nas publicações inicialmente identificadas. As bases eletrônicas pesquisadas foram literatura internacional em Ciências da Saúde (MEDLINE), Scientific Electronic Library Online (SciELO) e Biblioteca Virtual em Saúde do Ministério da Saúde (BVS). Foram utilizadas as palavras-chave transexualidade, transexuais, sexo e atendimento odontológico.

\section{Revisão bibliográfica}

A transgressão dos direitos humanos básicos no atendimento aos Gays, Lésbicas, Bissexuais, Travestis e Transexuais (LGBTTT), através de segregações, preconceitos, marginalização social, dentre outros por diversos grupos sociais, inclusive por profissionais de saúde, motivaram a formulação e criação de políticas públicas de saúde específicas para este público visando garantir a igualdade no atendimento. Questões culturais vindas do padrão heterossexual amplamente aceito socialmente podem influenciar no modo como os profissionais de saúde atendem a essa população e assim erguer uma barreira impedindo o acesso aos serviços de saúde pelo usuário LGBTTT (Cardoso \& Ferro, 2012).

Com a Constituição Federal de 1988 a nova carta constitucional passou a promulgar a saúde como direito do cidadão e dever do Estado, estabelecendo, assim, o sistema público de saúde, transformando a saúde pública no Brasil (Paiva; Teixeira, 2014). É então criada em 1990 a Lei 8.080, que regulamenta o Sistema Único de Saúde (SUS) que é formado por todos os serviços de saúde prestados sob administração do Poder Público, cabendo à iniciativa privada a complementaridade dos serviços (Silva, 2019). 
A formação do sistema de saúde é acompanhada de uma nova concepção de saúde que se relaciona com a qualidade de vida da população e não apenas com a ausência de doenças, como o conceito anterior. Previamente a construção do SUS, apenas possuía direito de acesso à saúde pública, as/os cidadãs/os que contribuíssem com a previdência social, ou seja, apenas os trabalhadores com carteira de trabalho assinada (Brasil, 2000). A ênfase do SUS está sob o âmbito municipal, devendo ser desenvolvida as principais ações e serviços segundo os sistemas municipais. A responsabilidade da gestão é dos municípios, estados, Distrito federal e da União, esta integrada a lei, o direito de participação social expressa pelos Conselhos de Saúde nos três níveis de governo e as Conferências de Saúde com paridade governamental e não governamental, uma que a organização da rede do SUS se dá de forma regionalizada e com nível de complexidade crescente (Silva, 2019).

A regionalização e a hierarquização da rede, das ações e dos serviços de saúde é referente às atribuições no que diz respeito as/os gestoras/es municipais e estaduais, que com seus recursos devem garantir a eficiência, efetividade e eficácia dos serviços de saúde disponibilizados pelo SUS (Brasil, 2000).

A Portaria $\mathrm{n}^{\circ}$ 2.803, de 19 de novembro de 2013 do Ministério da Saúde, redefine e amplia o Processo Transexualizador no Sistema Único de Saúde (SUS) dispõe sobre os procedimentos que são disponibilizados pela rede pública. São eles: Acompanhamento da/o usuária/o no processo transexualizador exclusivo nas etapas do pré e pós-operatório; Tratamento hormonal; Redesignação sexual no sexo masculino; Cirurgia de cordas vocais; Tratamento hormonal preparatório para cirurgia de redesignação sexual; Mastectomia simples bilateral; Histerectomia; Cirurgias complementares de redesignação sexual; Acompanhamento de usuária/o no Processo Transexualizador exclusivamente para atendimento clínico; Plástica mamária reconstrutiva incluindo prótese mamária. Convém destacar que o procedimento cirúrgico de neofaloplastia (transgenitalização) não está incluso por, segundo o Ministério da Saúde, ainda não apresentar um resultado satisfatório (Brasil, 2013).

Pessoas transexuais possuem a necessidade de adequarem seu corpo a sua identidade psíquica, podendo recorrer a procedimentos tais como tratamentos hormonais ou cirúrgicos. Não raro, há as/os transexuais que optam por não recorrer a métodos cirúrgicos (Galli et al., 2013). Desta forma, um homem transexual ou Female to Male (FTM) é o homem que possui sexo biológico feminino, porém se identifica com o gênero masculino. De mesma forma, uma mulher transexual ou Male to Female (MTF) apresenta o sexo biológico masculino, mas identifica-se como mulher (Brasil, 2016)

Haja vista as necessidades de saúde da população de Lésbicas, Gays, Bissexuais, Travestis e Transexuais (LGBT), em dezembro de 2011, o Ministério da Saúde do Brasil, implantou a Portaria no 2.836 que visa incluir no atendimento ao SUS, a Política Nacional de Saúde Integral LGBT. 24 A Portaria possui 24 objetivos específicos, os quais reforçam os princípios do SUS, anteriormente citados, com foco na saúde LGBTI+; ampliação de acesso; difundir dados e indicadores; promover o respeito em âmbito de saúde pública e fortalecer serviços para essa população (Brasil, 2011). Define nove diretrizes, as quais garantem o respeito aos direitos humanos bem como visam contribuir para a eliminação de preconceitos na sociedade e em âmbito de serviços de saúde; inclusão social para implementação de novos serviços e políticas e produção de novas tecnologias voltadas para a área de conhecimentos sobre LGBTI+ (Brasil, 2011).

É notável a ausência de travestis e transexuais em serviços de saúde, principalmente na atenção básica, serviço o qual deveria ser a porta de entrada para os serviços de saúde, resultando em um fato alarmante. A consequência é a segregação do grupo social para serviços específicos, com foco nos centros especializados no processo trasexualizador, comumente entendidos como locais com profissionais treinados e capacitados para receber e acolher essa população (Silva, et al., 2017).

Com a dificuldade no acesso por meio do SUS, grande parte da população transexual recorre a outros métodos de iniciar suas transformações corporais, a grande maioria sem acompanhamento de equipe de saúde, acarretando agravos à saúde, por meio de procedimentos clandestinos. (Rocon, et al., 2019). 
Além do forte impedimento que existe para as/os transexuais procurarem a serviços de saúde por conta da prática preconceituosa realizada por profissionais destes locais, fazendo com que a população transexual evite a busca de tratamento de saúde, e, levando em consideração a condição de vulnerabilidade social destes, pode resultar em adoecimento e até a morte (Rocon, et al., 2017).

Isso posto, respeitar a forma como o indivíduo transexual quer ser chamado, é uma maneira de concretizar o princípio de integralidade e, deste modo, criar uma abertura de criação de vínculo entre usuário e profissional de saúde, permitindo que esse paciente permaneça em contato com o serviço de saúde, diminuindo os índices de evasão. (Monteiro \& Brigeiro, 2019).

De acordo com Cassalha et al. (2020), a discriminação de transgêneros já ocorre na recepção devido a tratamentos que menosprezam o paciente, gerando uma série de situações vexatórias, o que afasta cada vez mais esse grupo de buscar auxílio da saúde. Podendo levar esses pacientes a obterem uma série de problemas irreversíveis e até mesmo letais, uma vez que muitas pessoas trans vivem em situação de vulnerabilidade social.

Isso posto, segundo Monteiro e Brigueiro et al., o primeiro passo que o Cirurgião-dentista deve tomar para atender de forma correta esse grupo é questionar ao paciente transexual qual o nome e pronome que ele prefere ser chamado, e sempre anotar seu nome social no prontuário. Desta forma, é dever do profissional se aperfeiçoar em relação a questões de gênero e sexualidade a fim de prevenir situações e propagações de preconceito, além de que o profissional deve ser contribuinte com as mudanças sociais atuais, mantendo-se atualizado para informar os direitos dessa população.

Portanto, segundo Ferreira et al deve garantir isonomia e um atendimento universal todos, pois espera-se do Cirurgião dentista, como profissional da saúde, que esse tenha, além dos conhecimentos técnicos o conhecimento da realidade social e atendimento humanitário, para assim enxergar de forma multidisciplinar se o paciente necessita de tratamento psicológico através da anamnese minuciosa, com perguntas detalhadas, evitando então práticas que levam à negação das diferenças individuais.

\section{Conclusão}

Deve existem uma prestação de serviço digna para os cuidados específicos a comunidade transexual. É importante o incremento de discussões e debates no meio dos profissionais de saúde para buscar formas de tornar o atendimento mais acolhedor e igualitário para a toda a população, visto que o profissional precisa ser urgentemente treinado para tratar os pacientes da forma preferencial deles, em virtude do acesso a saúde desse grupo ser muito limitado devido ao posicionamento da equipe de saúde mal preparada. Ainda, o Cirurgião-dentista, tem um papel imprescindível no acolhimento de transexuais e travestis para a criação de um vínculo isento de preconceitos, garantindo assim, um atendimento universal e humanitário.

Desse modo, recomenda-se que essa revisão possa contribuir na aplicabilidade do entendimento que torna indispensável que é dever de todos respeitar os direitos dessa população, independente de opiniões pessoais.

\section{Referências}

Albuquerque G. A. et al. (2013). Homossexualidade e o direito à saúde: Um desafio para as políticas públicas no Brasil. Saúde em Debate. Rio de Janeiro, 37(98), 516-524.

Aran M, et al. (2009). Transexualidade e saúde pública no Brasil. Ciênc. saúde coletiva, 14(4), 1141- 1149.

Aran M. (2006). A transexualidade e a gramática normativa do sistema sexo-gênero. Ágora, 9(1), 49-63.

Athayde A. V. L. (2001). Transexualismo masculino. Arq Bras Endocrinol Metab, 45(4), 407-414.

Brasil. (2000). Ministério da Saúde. Secretaria Executiva. SUS princípios e conquistas.

Brasil. (2003) Ministério da Saúde. Portaria nº 2.836, de 1 de dezembro de 2011. Diário Oficial da República Federativa do Brasil. Dez. 2011. 
Research, Society and Development, v. 10, n. 13, e587101321553, 2021

(CC BY 4.0) | ISSN 2525-3409 | DOI: http://dx.doi.org/10.33448/rsd-v10i13.21553

Brasil. (2013). Ministério da Saúde. Gabinete do Ministro. Portaria n ${ }^{\circ}$ 2.803, de 19 de novembro de 2013. Redefine e amplia o Processo Transexualizador no Sistema Único de Saúde (SUS). Diário Oficial da União, Brasília, DF, p. 25.

Brasil. (2016). Conselho Nacional de Saúde. Resolução nº 510.

Brasil. (2017). O Ministério Público e os direitos de LGBT: conceitos e legislação / Procuradoria Federal dos Direitos do Cidadão. Ministério Público do Estado do Ceará. MPF.

Cardoso, Michelle Rodrigues e Ferro, Luís Felipe. (2012). Saúde e população LGBT: demandas e especificidades em questão. Psicologia: Ciência e Profissão. $32(3)$.

Cassalha $\mathrm{O}$, et al. (2020) Vivências de pessoas transgêneras no atendimento à saúde: metassíntese qualitativa. Research, Society and Development, 9(10).

Ferreira K, et al. (2020). Gênero e Odontologia: Um relato de experiência. Revista Da Faculdade De Odontologia - UPF; 24(3), 417-421.

Galli R. A. et al. (2013). Corpos mutantes, mulheres intrigantes: transexualidade e cirurgia de redesignação sexual. Psicologia: Teoria e Pesquisa, Brasília, 29(4), 447-457.

Guimarães R et al. (2017). Assistência à saúde da população LGBT em uma capital brasileira: o que dizem os Agentes Comunitários de Saúde?. Tempus, actas de saúde colet, 11(1), 121-139.

Jesus J. G. (2012). Orientações sobre identidade de gênero: conceitos e termos. 2 ed. Brasília.

Monteiro S, \& Brigeiro M. (2019). Experiências de acesso de mulheres trans/travestis aos serviços de saúde: avanços, limites e tensões. Cadernos de Saúde Pública, 35 .

Paiva C. H. A., \& Teixeira L. A. (2014). Reforma sanitária e a criação do Sistema Único de Saúde: notas sobre contextos e autores. Hist. cienc. saudeManguinhos, 21(1), 15-36.

Rocon P. C., et al. (2017). O que esperam pessoas trans do Sistema Único de Saúde? Interface - Comunicação, Saúde, Educação, 22(64), 43-53.

Rocon P. C., et al. (2019). Desafios enfrentados por pessoas trans para acessar o processo transexualizador do Sistema Único de Saúde. InterfaceComunicação, Saúde, Educação, 23.

Rocon P. C., et al. (2020). Vidas após a cirurgia de redesignação sexual: sentidos produzidos para gênero e transexualidade. Scielo saúde pública.

Saadeh A. (2004). Transtorno de identidade sexual: um estudo psicopatológico de transexualismo masculino e feminino. Universidade de São Paulo,

Santos E, et al. (2020). Dificuldades enfrentadas por lésbicas, gays, bissexuais, travestis, transexuais e transgêneros ao atendimento no Programa de Saúde da Família. Research, Society and Development, v. 9(9).

Silva J. B. (2019). Participação social e comunicação: disputas e negociações no Conselho Municipal de Saúde do Rio de Janeiro. 2019. 32 f. Trabalho de Conclusão de Curso (Especialização em Comunicação em Saúde) - Instituto de Comunicação e Informação Científica e Tecnológica em Saúde, Fundação Oswaldo Cruz, Rio de Janeiro.

Silva L. K. M, et al. (2017). Uso do nome social no Sistema Único de Saúde: elementos para o debate sobre a assistência prestada a travestis e transexuais. Physis, 27(3), 835-846.

Silva M. E. A. (2019). A divisão do esporte deve ser separada por sexo ou gênero. REDOC, 3(1), 236-249.

Trindade C. A., et al. (2019). Posicionamento Conjunto. Medicina Diagnóstica inclusiva: cuidando de pacientes transgênero. Sociedade Brasileira de Patologia Clínica/Medicina Laboratorial (SBPC/ML). Sociedade Brasileira de Endocrinologia e Metabologia (SBEM). Colégio Brasileiro de Radiologia e Diagnóstico por Imagem (CBR). 